\title{
Sodium-glucose Cotransporter 2 Inhibitors' Rise to the Backbone of Heart Failure Management: A Clinical Review
}

\author{
Namit Rohant ${ }^{1}$ and Jamie LW Kennedy² \\ 1. Advanced Heart Failure and Transplant Cardiology, University of California, San Francisco, CA, USA; 2. Inova Heart and Vascular Institute, \\ Falls Church, VA, USA
}

DOI: https://doi.org/10.17925/HI.2021.15.1.42

S odium-glucose cotransporter (SGLT) 2 inhibitors, or gliflozins, have quickly risen to prominence within the cardiovascular field due to their substantial benefit in the management of heart failure with reduced ejection fraction (HFrEF). SGLT channels are present throughout the body in various isoforms, but SGLT1 and SGLT2 have been the centre of medical investigation due to known genetic mutations. SGLT2 plays a major role in renal re-absorption of glucose, prompting the development of SGLT2 inhibitors to promote glycosuria and aid in diabetes management. The United States Food and Drug Administration requires evaluation of new antidiabetic medications for cardiovascular safety, prompting several randomized controlled trials of SGLT2 inhibitors over the past 5 years. These initial trials demonstrated superiority in cardiovascular outcomes with SGLT2 inhibitor use and suggested particular benefit in heart failure (HF) outcomes, prompting further study of their mechanisms. Subsequent SGLT2 inhibitor studies have demonstrated reductions in HF hospitalizations and cardiovascular mortality in patients with HFrEF, regardless of the presence of diabetes mellitus. In this review, we discuss the mechanism of action and major clinical trial results that have propelled SGLT2 inhibitors into a key role for patients with HFrEF.

\section{Keywords}

Sodium-glucose cotransporter (SGLT) 2 inhibitors, SGLT1, heart failure, guideline-directed medical therapy (GDMT), dapagliflozin, empagliflozin, canagliflozin, ertugliflozin, sotagliflozin, phlorizin

Disclosure: Namit Rohant and Jamie LW Kennedy have no financial or non-financial relationships or activities to declare in relation to this article.

Review process: Double-blind peer review.

Compliance with ethics: This study involves a review of the literature and did not involve any studies with human or animal subjects performed by any of the authors.

Authorship: The named authors meet the International. Committee of Medical Journal Editors (ICMJE) criteria

for authorship of this manuscript, take responsibility for the integrity of the work as a whole, and have given final approval for the version to be published.

Access: This article is freely accessible at touchCARDIO.com. (C) Touch Medical Media 2021

Received: 1 February 2021

Accepted: 25 May 2021

Published online: 15 July 2021

Citation: Heart International. 2021;15(1):42-8

Corresponding author: Jamie LW Kennedy, Inova Heart and Vascular Institute, 3300 Gallows Rd, Falls Church, VA 22042 USA. E: jamie.kennedy@inova.org

Support: No funding was received in

the publication of this article.
Heart failure (HF) is an increasingly prevalent clinical syndrome resulting from a variety of disease processes impeding the heart's ability to effectively circulate blood. With advances in care and a steadily growing and aging population, HF management is becoming a foremost priority in developed nations like the USA, where HF is expected to affect more than $3 \%$ of the overall population or 8.5 million people by $2030 .{ }^{1}$ This increase in population burden directly translates into patient morbidity and mortality, as well as the significant economic costs of delivering healthcare, projected to be approximately $\$ 70$ billion for the USA by $2030 .{ }^{2}$

The syndrome of HF was first described by several ancient civilizations. A greater understanding of HF has steadily developed over the centuries, and in the early 20th century, modern therapeutics in the form of diuretic medications established a treatment approach for $\mathrm{HF}^{3}$ Guideline-directed medical therapy (GDMT), medications proven in clinical trials to reduce morbidity and mortality, started in 1986 when the V-HeFT trial demonstrated a mortality benefit with the use of isosorbide dinitrate and hydralazine compared with prazosin and placebo. ${ }^{4}$ Shortly afterwards in 1987, angiotensin-converting enzyme inhibitors (ACE-I), specifically enalapril, presented mortality benefits in the CONSENSUS trial. ${ }^{5}$

Subsequent GDMT focused on mortality benefits derived from neuro-hormonal blockade in the form of beta blockers; ACE-I, angiotensin-2-receptor blockers (ARB), and angiotensin receptor-neprilysin inhibitors (ARNI); and mineralocorticoid receptor antagonists (MRA). Additionally, devices such as internal cardioverter defibrillators and biventricular pacemakers have been added to the arsenal of tools to reduce morbidity and mortality. However, the burden of HF remains substantial, prompting ongoing efforts to identify novel therapeutic targets. While several new treatment options have modest benefits or limited applicability, sodium-glucose cotransporter 2 (SGLT) 2 inhibitors have exhibited significant benefits proportional to neurohormonal blockade GDMT.

\section{Sodium-glucose cotransporter mechanism of action and initial development}

SGLT proteins exist in many tissues throughout the human body in several isoforms and operate in active glucose uptake into cells and as water channels. ${ }^{6.7}$ Of the several isoforms, SGLT1 and SGLT2 have been the focus of modern medicine due to known genetic diseases arising from spontaneous mutations in S/C5a1 and S/C5a2 genes, respectively. ${ }^{7}$ SGLT1 is located in the kidneys, intestines, liver, lungs, brain and heart, while SGLT2 predominates the S1 and S2 segments of the apical brush border membrane of the renal proximal convoluted tubules. ${ }^{8}$ 
Table 1: Overview of sodium-glucose cotransporter 2 inhibitor diabetes trials ${ }^{19-27}$

\begin{tabular}{|c|c|c|c|c|c|}
\hline Trial and date & Medication and dose(s) & HF patients & CAD patients & Primary cardiac findings & Major adverse events \\
\hline $\begin{array}{l}\text { EMPA-REG (NCT01131676), } \\
\text { 201519,20 }\end{array}$ & Empagliflozin 10/25 mg & $\begin{array}{l}9.9 \% \mathrm{~T} \\
10.5 \% \mathrm{P}\end{array}$ & $\begin{array}{l}75.6 \% \mathrm{~T} \\
75.6 \% \mathrm{P}\end{array}$ & $\begin{array}{l}\text { Superior in MACE; reduced HF } \\
\text { hospitalizations }\end{array}$ & Increased genital infections \\
\hline $\begin{array}{l}\text { CANVAS } \\
\text { Program (NCT01032639, } \\
\text { NCT01989754), } \\
201721-23\end{array}$ & Canagliflozin 100/300 mg & $\begin{array}{l}13.9 \% \mathrm{~T} \\
15.1 \% \mathrm{P}\end{array}$ & $\begin{array}{l}55.8 \% \mathrm{~T} \\
57.2 \% \mathrm{P}\end{array}$ & $\begin{array}{l}\text { Superior in MACE; } \\
\text { non-significant secondary outcomes in } \\
\text { CV findings }\end{array}$ & $\begin{array}{l}\text { Increased amputations and } \\
\text { genital infections }\end{array}$ \\
\hline $\begin{array}{l}\text { DECLARE-TIMI } 58 \\
\text { (NCT01730534), } \\
2019^{24,25}\end{array}$ & Dapagliflozin $10 \mathrm{mg}$ & $\begin{array}{l}9.9 \% \mathrm{~T} \\
10.2 \% \mathrm{P}\end{array}$ & $\begin{array}{l}32.9 \% \mathrm{~T} \\
33.0 \% \mathrm{P}\end{array}$ & $\begin{array}{l}\text { Non-inferior in MACE; superior for } \\
\text { composite CV death or HF hospitalization }\end{array}$ & $\begin{array}{l}\text { Increased DKA and genital } \\
\text { infection }\end{array}$ \\
\hline $\begin{array}{l}\text { VERTIS CV (NCT01986881), } \\
202026,27\end{array}$ & Ertugliflozin 5/15 mg & $\begin{array}{l}23.4 \% \mathrm{~T} \\
24.5 \% \mathrm{P}\end{array}$ & $\begin{array}{l}75.4 \% \mathrm{~T} \\
76.9 \% \mathrm{P}\end{array}$ & Non-inferior in MACE & $\begin{array}{l}\text { Increased urinary tract and } \\
\text { genital infections }\end{array}$ \\
\hline
\end{tabular}

$C A D=$ coronary artery disease; $C V=$ cardiovascular; $D K A=$ diabetic ketoacidosis; $H F=$ heart failure; $M A C E=$ major adverse cardiovascular events; $P=$ placebo arm;

$T=$ treatment arm.

Both SGLT1 and SGLT2 actively facilitate reabsorption of glucose by cotransport of sodium down its electrochemical gradient into the cell. However, the coupling of sodium and glucose for the two channels is variable, 2:1 for SGLT1 and 1:1 for SGLT2. ${ }^{9}$ With these dynamics, SGLT2 reabsorbs approximately $90 \%$ of the filtered glucose in the kidneys, with SGLT1 in the S3 segment reabsorbing the remainder. ${ }^{10}$ The absolute percentage is variable due to the sequential nature of glucose reabsorption in the kidneys.

Owing to their fundamental role in renal glucose reabsorption, inhibitors for SGLT1 and SGLT2 were developed over the past decade as a novel class of antidiabetic medications. Phlorizin is a botanical compound that was first isolated from fruit trees in 1835 and has since benefitted pharmaceutical research. Its primary mechanism of action is avidly inhibiting SGLT1 and SGLT2, resulting in renal glycosuria while avoiding hypoglycaemia. ${ }^{11}$ Although employing this characteristic for diabetes management is ideal, phlorizin is not fitting as clinical pharmacotherapy because it is poorly absorbed from the gastrointestinal tract." However, phlorizin's potential led to the development of SGLT inhibitors, with a particular focus on SGLT2 inhibitors, which serve as the main site for glucose reabsorption.

As SGLT2 inhibitors were developed and studied as antiglycaemic therapy, researchers uncovered certain characteristics from this class of medications. The pharmacodynamics of SGLT2 inhibitors are not affected by other diabetes medications. ${ }^{12}$ The onset of action is approximately 1-2 hours, and the offset of action, based on dose, is roughly 24-48 hours. ${ }^{13}$ Additionally, the fractional excretion of glucose achieved by SGLT2 inhibition remains constant, despite patient size and degree of insulin resistance. With these features, the average glucose reabsorption prevented by SGLT2 inhibitors is in the range of $30-50 \%$, driven by the intrinsic potency of the particular SGLT2 inhibitor. ${ }^{6}$

The expanding role of SGLT2 inhibitors from diabetes treatment to HF management is fortuitous. In 2007, Nissen and Wolski published a meta-analysis demonstrating a significantly increased risk of myocardial infarction associated with the use of rosiglitazone. ${ }^{14}$ In response to this study, the US Food and Drug Administration (FDA) has required assessment of cardiovascular outcomes and safety for new diabetes medications..$^{15}$ Since then, randomized controlled trials with diabetes medications have been designed as non-inferiority studies compared to placebo, with a primary outcome composite of major adverse cardiovascular events (MACE) combining cardiovascular mortality, non-fatal myocardial infarction and non-fatal strokes. ${ }^{16}$

\section{Initial sodium-glucose cotransporter 2 inhibitor trials for diabetes management indicating cardiovascular benefit}

In the past decade, investigators have tested a number of different SGLT inhibitors in large-scale randomized clinical trials to evaluate their efficacy in diabetes management and to observe the cardiovascular safety profile. The SGLT2 inhibitors, or gliflozins, in clinical use possess varying affinities for the SGLT2 cotransporter. Empagliflozin is highly selective for SGLT2 with SGLT2:SGLT1 binding of approximately 2,700:1; whereas canagliflozin, with the lowest selectivity, has only a 160-fold higher affinity for SGLT2. Dapagliflozin, one of the more frequently used SGLT2 inhibitors for HF, has a >1,200-fold affinity for SGLT2 over SGLT1. ${ }^{17}$ Conversely, sotagliflozin is essentially a non-selective SGLT inhibitor, binding SGLT2:SGLT1 with a 20:1 ratio. ${ }^{18}$ Discussed below are the major initial trials examining cardiovascular safety with SGLT2 inhibitors, which are also summarized in Table 1.19-27

In late 2015, Zinman et al., in their EMPA-REG OUTCOME trial (NCT01131676), published the first randomized controlled trial of empagliflozin and its cardiovascular safety after FDA approval. ${ }^{1920}$ This trial compared the effects of cardiovascular morbidity and mortality in patients with type 2 diabetes mellitus and established atherosclerotic cardiovascular disease on standard-of-care therapy; approximately $10 \%$ of patients were diagnosed with HF at enrolment. A total of 7,028 patients in 42 countries were randomly assigned to receive empagliflozin $10 \mathrm{mg}$ or $25 \mathrm{mg}$ daily or placebo in a 1:1:1 fashion. For the first 12 weeks, other antiglycaemic therapy could not be adjusted, but after that period, investigators were encouraged to augment therapy if needed to improve glycaemic control. The primary outcome was a MACE composite, as required by the FDA, and the secondary outcome was a composite of the primary outcome and hospitalization for unstable angina. The primary outcome occurred in a significantly lower percentage of patients on empagliflozin (10.5\%) compared with placebo (12.1\%) over a median follow-up of 3.1 years, meeting criteria for superiority and driven by a reduction in cardiovascular death. The secondary outcome met criteria for non-inferiority, occurring in $12.8 \%$ treated with empagliflozin compared to $14.3 \%$ on placebo. Other key findings included reduced all-cause mortality ( 5.7 versus $8.3 \%$ ) and HF hospitalizations (2.7 versus $4.1 \%$ ) with empagliflozin. Adverse events were similar between the groups except for more genital infections occurring in the empagliflozin groups.

The CANVAS Program published its results in 2017, examining the safety of canagliflozin in patients with diabetes with established, or high risk for, atherosclerotic cardiovascular disease. ${ }^{21}$ At enrolment, $14.4 \%$ of patients 
carried a diagnosis of HF. This study combined data from two related trials. An initial trial (CANVAS; NCT01032629) was introduced in December 2009, prior to FDA approval for the use of the medication. ${ }^{22}$ After canagliflozin became the first SGLT2 inhibitor granted FDA approval in March 2013, a second trial (CANVAS-Renal [CANVAS-R; NCT01989754]) was designed in a similar fashion to meet the revised post-approval FDA requirement. ${ }^{23}$ The data from the two trials were pooled to examine a primary outcome of MACE and secondary outcomes of death from any cause, cardiovascular death, albuminuria, and a composite of cardiovascular death and HF hospitalizations. A total of 10,142 patients were involved (4,330 in CANVAS and 5,812 in CANVAS-R), 9,734 of which completed the trial. After a 2-week run-in period, participants were randomized to a 1:1:1 ratio in CANVAS, to receive either canagliflozin $100 \mathrm{mg}$ or $300 \mathrm{mg}$ daily, or placebo. The participants in CANVAS-R were randomized 1:1 to canagliflozin $100 \mathrm{mg}$ daily or placebo, with an option to increase canagliflozin to $300 \mathrm{mg}$ daily after 13 weeks (of which, $71.4 \%$ participants in the canagliflozin group did). Canagliflozin demonstrated superiority for the primary outcome: 26.9 events per 1,000 patient-years compared with 31.5 events for placebo. The secondary outcomes did not meet statistical significance; however, there was a signal towards reduction of cardiovascular death and HF hospitalizations ( 16.3 versus 20.8 events per 1,000 patient-years) and albuminuria (89.4 versus 128.7 events per 1,000 patient-years) with canagliflozin treatment. A new finding of increased amputations, especially at the toe or metatarsal level, was noticed with canagliflozin use, but other adverse effect rates were as expected. ${ }^{21}$

Dapagliflozin was the first SGLT2 inhibitor approved in the world for clinical use by the European Union in 2012. Its cardiovascular safety outcomes in patients with diabetes with established, or at high risk for, atherosclerotic cardiovascular disease were reported in 2019 by the DECLARE-TIMI 58 investigators (NCT01730534);24,25 approximately $10 \%$ of patients were diagnosed with HF at enrolment. As per prior trials, the primary safety outcome was MACE. There were also primary efficacy outcomes of MACE and a composite of cardiovascular death or HF hospitalizations, building on the positive results of the EMPA-REG OUTCOME trial..$^{19,20}$ Secondary efficacy outcomes included a renal composite, as well as death from any cause. A total of 25,698 patients enrolled initially, of which, 17,160 completed a 4- to 8-week run-in period with placebo alone and then were randomly assigned 1:1 to dapagliflozin $10 \mathrm{mg}$ daily or placebo. Dapagliflozin was non-inferior to placebo for the primary safety outcome of MACE, $8.8 \%$ for dapagliflozin compared with $9.4 \%$ for placebo over a median of 4.2 years. For the primary efficacy outcomes, dapagliflozin was not superior to placebo in terms of MACE, but did meet criteria for superiority for the composite of cardiovascular death or HF hospitalization (4.9\% versus $5.8 \%$ in placebo). of note, this composite was fundamentally driven by a reduction in HF hospitalization, as there was no difference in cardiovascular death between the two groups. Furthermore, there were significantly fewer renal events in the dapagliflozin group. Overall safety and adverse events trended towards higher rates of diabetic ketoacidosis and genital infections in the dapagliflozin group, compared with the placebo. ${ }^{24}$

The VERTIS CV trial (NCT01986881), published in 2020, describes cardiovascular outcomes for the more-recently approved ertugliflozin. 26,27 A total of 8,238 patients with type 2 diabetes mellitus and established atherosclerotic cardiovascular disease were randomized into groups receiving ertugliflozin $5 \mathrm{mg}$ or $15 \mathrm{mg}$ daily or placebo on a 1:1:1 ratio; $23.7 \%$ of patients had a history of HF. The ertugliflozin groups were combined for outcomes analysis. Ertugliflozin was non-inferior to placebo in terms of the primary outcome of MACE; $11.9 \%$ for both, over a median follow-up of 3.5 years. The secondary outcome of cardiovascular death or HF hospitalization was not significantly different between the groups. Adverse events were similar to other SGLT2 inhibitors, with higher urinary and genital infections and numerically higher, but not significantly higher, rates of amputations and diabetic ketoacidosis. ${ }^{26}$

These initial studies were designed, based on the rosiglitazone experience, to detect increased risk of myocardial infarction and other atherosclerotic events, and enrolled patients at risk for such events; HF was not initially considered a priority. Furthermore, patients with chronic HF only comprised a small portion of the study populations and were not further characterized by functional class or ejection fraction. Interestingly, the earlier trials (EMPA-REG and CANVAS) displayed superiority in MACE, but the subsequent trials did not. Differences among the trial populations likely contributed to this variation; the prevalence of coronary disease ranged from 33\% in DECLARE-TIME 58, to $76 \%$ in both EMPA-REG and VERTIS CV. A variety of other factors may also have contributed, including trial design variances, differences in SGLT2 inhibitors, more intensive secondary therapy for MACE, and others. It is interesting that the benefits noted were independent of the glycated haemoglobin (HbA1C)-lowering effects of the medications. Outside of these, a number of other SGLT2 inhibitors have been developed and are being tested globally, such as ipragliflozin (NCT02479399), luseogliflozin (NCT02500186), tofogliflozin (NCT02201004) and remogliflozin (NCT02537470). ${ }^{28-31}$

\section{Presumed cardiovascular mechanism of action and benefit of sodium-glucose cotransporter 2 inhibition}

SGLT inhibition has been postulated to be beneficial in cardiovascular disease through a variety of mechanisms. The obvious benefit of improved diabetes control aside, the glycosuria also results in weight loss from caloric wasting, and waist circumference reduction, leading to a reduction in insulin resistance; however, the weight loss from SGLT2 inhibitors is modest, averaging $1.5-2 \mathrm{~kg} .{ }^{32}$ This may also be related to suggestive reductions in epicardial adipose tissue, and hence, coronary atherosclerosis; though one would expect to realize the benefits of this mechanism over years. ${ }^{33}$ Moreover, there are known overwhelming changes in myocardial insulin signalling, secondary to generalized insulin resistance, resulting in myocardial dysfunction. This is surmised to be due to reduced insulin-mediated uptake and utilization of glucose for required contractility of the myocardial cells. It is believed that chronic pathologic conditions, such as HF, result in euglycaemic hyperinsulinaemia, prompting resistance in those without diabetes. ${ }^{34}$ Modulation of insulin resistance, and hence, myocardial insulin signalling, may be a potential benefit of SGLT2 inhibitors as well. ${ }^{34}$

By increasing urinary glucose and sodium excretion, there is accompanying osmotic diuresis, which results in decreased preload and augments the effects of other diuretics. One small study demonstrated a significant reduction in pulmonary capillary wedge pressure, but no improvement in cardiac index after 12 weeks of empagliflozin use. ${ }^{35}$ There are some suggestions, that with prolonged SGLT2 inhibition, energy dynamics undergo changes similar to those seen with aestivation. This physiologic state required to survive arid hot conditions promotes water-conservation systems and switches to fatty acid utilization and ketogenesis to counteract fuel loss in the form of glycosuria, ultimately enhancing myocardial energetics. ${ }^{36}$ There is evidence of improved erythropoiesis with SGLT2 inhibitor use, resulting in better end-organ oxygenation. ${ }^{37}$

Hyperuricemia is associated with increased risk of cardiovascular disease; SGLT2 inhibitors promote augmented uric acid excretion via 
Table 2: Overview of sodium-glucose cotransporter 2 inhibitor heart failure trials ${ }^{41-46}$

\begin{tabular}{|l|l|l|l|l|}
\hline $\begin{array}{l}\text { Trial and year } \\
\begin{array}{l}\text { DAPA-HF } \\
\text { (NCT03036124), } \\
201941,42\end{array}\end{array}$ & Dedication and dose & Diabetic patients & Primary outcome & \multicolumn{1}{|l}{ Major adverse events } \\
\hline $\begin{array}{l}\text { EMPEROR-Reduced } \\
\text { (NCT03057977), } \\
\text { 202043,44 }\end{array}$ & Empagliflozin $10 \mathrm{mg}$ & $50 \%$ in each arm & Reduction in HF hospitalization and CV death & Increased genital infections \\
\hline $\begin{array}{l}\text { SOLOIST-WHF } \\
\text { (NCT03521934), } \\
\text { 202145,46 }\end{array}$ & $\begin{array}{l}\text { Sotagliflozin* } \\
200 / 400 ~ m g\end{array}$ & $100 \%$ in each arm & $\begin{array}{l}\text { Reduction in CV deaths, HF hospitalizations, } \\
\text { and urgent HF visits }\end{array}$ & $\begin{array}{l}\text { Increased diarrhoea and incidence of } \\
\text { severe hypoglycaemia }\end{array}$ \\
\hline
\end{tabular}

All primary cardiac findings were statistically significant. Major adverse events are compared to placebo.

*Sotagliflozin is a nonspecific SGLT1/SGLT2 inhibitor.

$\mathrm{CV}=$ cardiovascular; $\mathrm{HF}=$ heart failure.

Figure 1: Cumulative patient populations and major endpoints from the three major sodium-glucose cotransporter 2 inhibitor heart failure trials

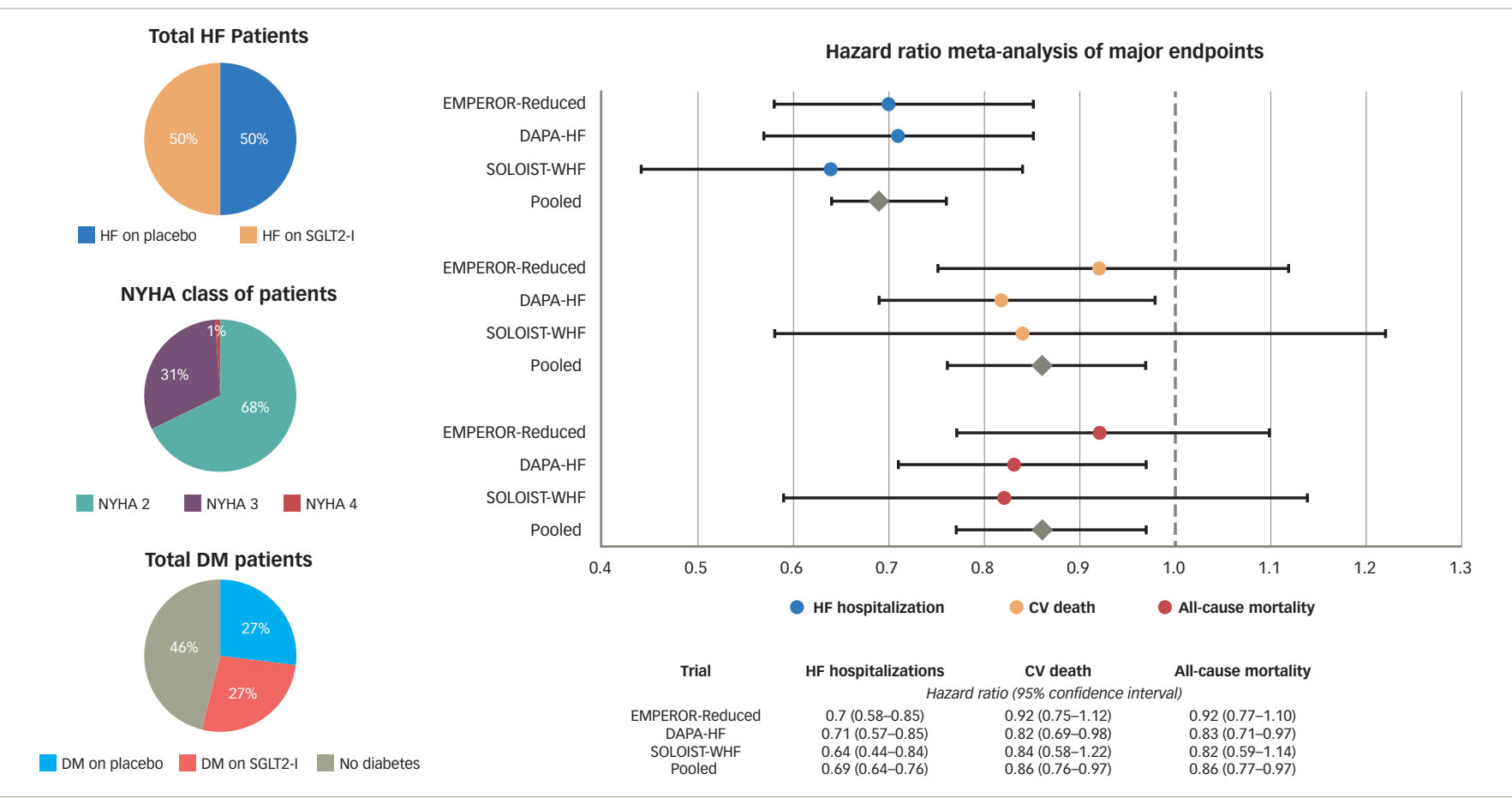

The panels on the left indicate percentage of total patients in those respective populations across the three major SGLT2 inhibitor heart failure trials. The figure on the right graphically shows the meta-analysis of the individual trial endpoints of HF hospitalizations, CV deaths and all-cause mortality, as well as a pooled hazard ratio for each of those endpoints calculated using a fixed-effect meta-analysis method. The table at the bottom numerically displays the hazard ratios and the $95 \%$ confidence intervals for those endpoints.

CV = cardiovascular; $D M=$ diabetes mellitus; HF = heart failure; NYHA = New York Heart Association; SGLT2 = sodium-glucose cotransporter 2 inhibitor.

increased glucose excretion and inhibit its reabsorption via the GLUT9 isoform 2 transporters. ${ }^{38}$ Along with glycosuria, sodium excretion is an expected effect of SGLT inhibition, resulting in reduced systolic blood pressure. Hypertension has long been recognized as a major risk factor for the development of $\mathrm{HF}$, though, as with the epicardial adipose tissue discussion above, one would expect to realize this benefit after years of therapy. In addition, SGLT2 inhibitors have also been shown to reduce inflammation and high-sensitivity C-reactive protein (CRP) levels. ${ }^{39}$ Both the reduction in systolic blood pressure and high-sensitivity CRP are associated with reduced vascular resistance and arterial stiffness. ${ }^{39}$ All three of these parameters are known to result in improved cardiovascular outcomes. Further posited mechanisms that may be beneficial include desirable changes in myocardial metabolism and reduction in cardiac fibrosis. ${ }^{40}$ In summary, further study is needed to understand which of these mechanisms is responsible for the beneficial clinical effects of SGLT2 inhibitors.

\section{Sodium-glucose cotransporter 2 inhibitor trials for heart failure management}

After the initial randomized controlled trials revealed not only superior outcomes in MACE but also suggested reduced HF hospitalizations, several trials have been conducted to explore the benefits of SGLT2 inhibitors, specifically in patients with HF (Table $2^{41-46}$ and Figure 1). The DAPA-HF trial (NCT03036124) was published by McMurray et al. in November 2019.41,42 The HF inclusion criteria were an ejection fraction of $\leq 40 \%$, New York Heart Association (NYHA) functional class II-IV symptoms and a range of $\mathrm{N}$-terminal pro-B-type natriuretic peptide (NT-proBNP) levels based on last HF hospitalization and atrial arrhythmias. 
Figure 2: Comparison of relative risk reduction in landmark trials ${ }^{59-68}$

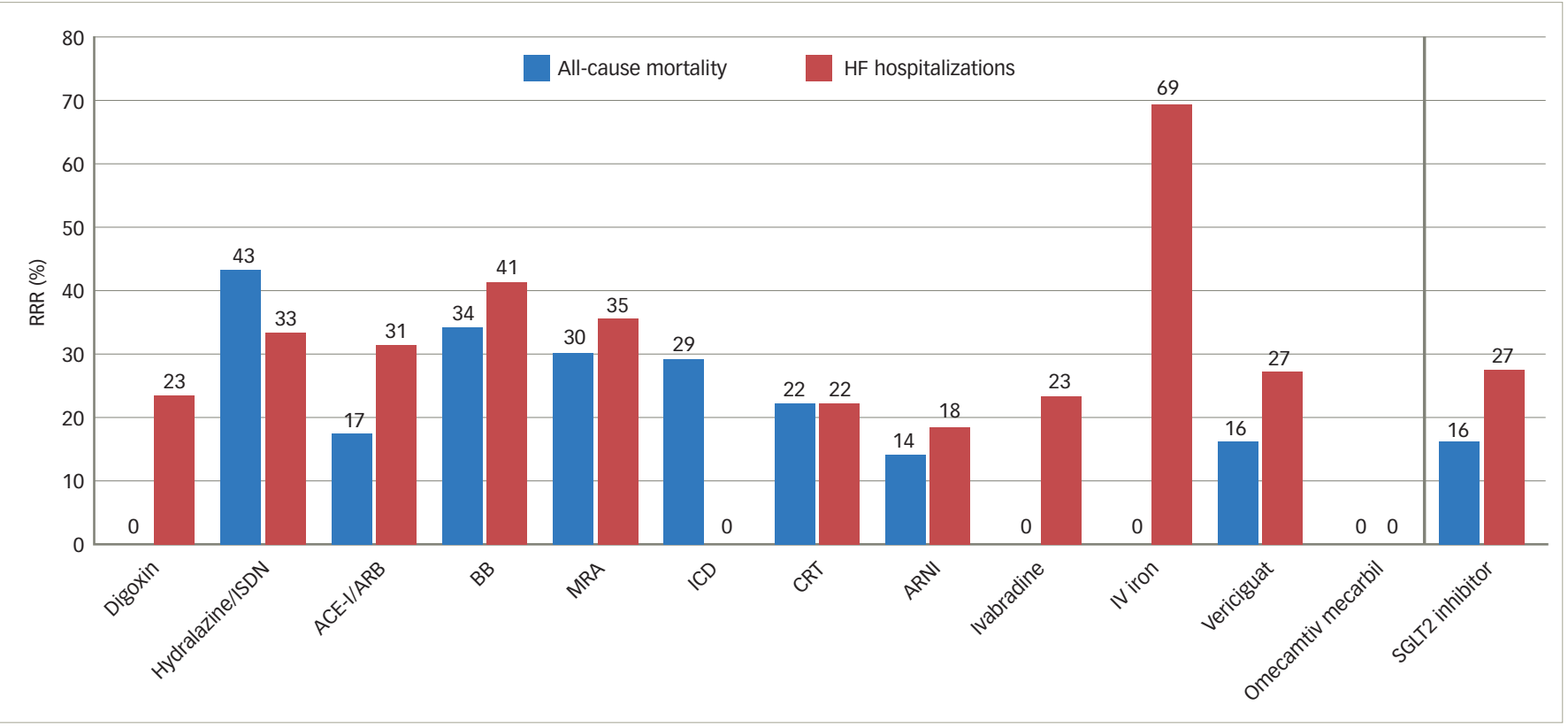

$A C E-I / A R B=$ angiotensin-converting enzyme inhibitor/angiotensin receptor blocker; $A R N I=$ angiotensin receptor neprilysin inhibitor; $B B=$ beta blocker; $C R T=c a r d i a c$ resynchronization therapy; $H F=$ heart failure; ICD = implantable cardioverter defibrillator; ISDN = isosorbide dinitrate; $I V=$ intravenous; $M R A=$ mineralocorticoid receptor antagonists; $R R R=$ relative risk reduction; SGLT2 = sodium-g/ucose cotransporter 2 inhibitor.

The use of ACE-I/ARB/ARNI and beta blockers was required unless contraindicated, MRA was encouraged, and implantable cardioverter defibrillators (ICDS) and cardiac resynchronization therapy (CRT) devices were required per guidelines. A diagnosis of diabetes mellitus was not required for enrolment; $41.8 \%$ of patients had diabetes. A total of 4,744 patients across 20 countries were randomly assigned to dapagliflozin $10 \mathrm{mg}$ daily or placebo. These patients were well-managed with $96 \%$ prescribed beta blockers, 84\% ACE-I/ARB, 10.7\% ARNI and 71\% MRA. Device utilization was less common: $26 \%$ had ICDS and $7 \%$ CRT devices, presumably due to variations in local guidelines for device implantation.

The primary outcome, a composite of worsening HF (hospitalization or urgent visit requiring intravenous therapy) or cardiovascular death, occurred in $16.3 \%$ of patients in the dapagliflozin group and $21.2 \%$ of patients in the placebo group over a median follow-up of 18.2 months, significant with $p<0.001$. Moreover, all three components significantly favoured the use of dapagliflozin, including in patients without diabetes. The secondary outcome, a composite of HF hospitalization or cardiovascular death, and Kansas City Cardiomyopathy Questionnaire (KCCQ) assessing HF symptoms, also favoured dapagliflozin use. Serious adverse events, such as volume depletion and renal events, were not significantly different between placebo and intervention arms; diabetic ketoacidosis and genital infections were more common in the dapagliflozin arm. ${ }^{41}$ Another dapagliflozin study, DEFINE-HF, a small-scale, short-term, randomized controlled trial performed by Nassif and colleagues, also showed significant improvement in KCCQ scores and HF symptoms, though the co-primary endpoint of improved 6-minute walk distance was not met. ${ }^{47}$

The EMPEROR-Reduced investigators performed a similar trial assessing the utility of empagliflozin for patients with HF with or without diabetes (NCT03057977).43,44 The HF inclusion criteria were similar to DAPA-HF, though the NT-proBNP levels were different. Nearly half of the patients had diabetes. Patients were assigned in a 1:1 ratio to receive either empagliflozin $10 \mathrm{mg}$ daily or placebo. The primary outcome was a composite of cardiovascular death or HF hospitalization. The key secondary outcomes were the occurrence of all adjudicated hospitalizations (first and recurrent) for $\mathrm{HF}$ and rate of decline in estimated glomerular filtration rate. A total of 3,730 patients were enrolled. Similar to DAPA-HF, beta blocker use was $95 \%$, ACE-I/ARB 70\%, ARNI 19\% and MRA 71\%, while cardiac devices were more common ( $31 \%$ had ICDS and $12 \%$ CRT devices). The primary outcome occurred in $19.4 \%$ of empagliflozin versus $24.7 \%$ of placebo patients over a median follow-up of 16 months ( $p<0.001)$, and was driven by reduction in HF hospitalizations. As previously seen, genital infections were more common with empagliflozin. Of particular interest, adverse events and safety concerns commonly appreciated with GDMT for HF, such as hypotension, renal dysfunction and electrolyte abnormalities, were not more common with empagliflozin use. ${ }^{43}$ In the EMPA-TROPISM trial (NCT03485222), 84 patients with HF with reduced ejection fraction (HFrEF) without diabetes were treated with empagliflozin $10 \mathrm{mg}$ daily for 6 months. ${ }^{48,49}$ Investigators found significantly reduced left ventricular (LV) end-systolic and diastolic volumes by cardiac magnetic resonance imaging, the primary endpoint, as well as increased LV ejection fraction (LVEF), reduced LV mass and spherical remodelling, increased peak oxygen consumption, and enhanced quality of life by KCCQ scores. ${ }^{48,49} \mathrm{~A}$ similar positive impact on reverse LV remodelling with empagliflozin use was detected in patients with HF and diabetes in the SUGAR-DM-HF trial (NCT03485092). ${ }^{50,51}$

The SOLOIST-WHF trial (NCT03521934), published in January 2021, examined the usefulness of the non-selective SGLT inhibitor, sotagliflozin, in patients with diabetes during a HF hospitalization with elevated BNP or NT-proBNP and requiring IV diuretic therapy. Importantly, there were no LVEF criteria.45,46 Patients enrolled were clinically stable, did not require supplemental oxygen or intravenous therapies and had systolic blood pressure $100 \mathrm{mmHg}$ or higher. A total of 1,222 patients $(79.1 \%$ with ejection fraction $<50 \%)$ were randomized to receive sotagliflozin $200 \mathrm{mg}$ daily (increased to $400 \mathrm{mg}$ daily depending on adverse reactions) or placebo before 
(48.8\% of participants) or within 3 days $(51.2 \%)$ of hospital discharge. GDMT was excellent; 92\% prescribed beta blockers, 64\% MRA, 83\% ACE-I/ARB and $17 \%$ ARNI. Because trial enrolment closed early after loss of funding, the primary outcome was changed to a composite of the total number of cardiovascular deaths, HF hospitalizations and urgent HF visits. Primary outcome analysis discovered sotagliflozin use to be favourable (51.0 versus 76.3 events per 100 patient-years, $p<0.001$ ), and was consistent across multiple pre-specified subgroups, including LVEF $\geq 50 \%$. The patients on sotagliflozin, compared with those on placebo, did suffer from increased diarrhoea (6.1\% versus $3.4 \%$ ) and severe hypoglycaemia (1.5\% versus $0.3 \%$ ). While this trial was limited due to loss of funding, the trial did suggest benefit of SGLT inhibition in managing recently decompensated HF and HF with preserved EF (HFpEF).

\section{The future of sodium-glucose cotransporter 2 inhibition and concluding remarks}

While the benefit of SGLT2 inhibition for diabetes management has long been theorized, improved cardiovascular outcomes were not expected. Several major studies involving thousands of patients have clearly shown the utility of SGLT2 inhibitors in improving cardiovascular outcomes, especially HF. Benefits have been seen with multiple SGLT2 inhibitors, suggesting a class effect. Ongoing and future studies (EMPERORPreserved [NCT03057951] for empagliflozin and DELIVER [NCT03619213] for dapagliflozin) are examining the potential benefit of SGLT2 inhibitors in HFpEF, building on the SOLOIST-WHF trial..$^{44-46,52}$ Moreover, the recently published studies such as the CREDENCE (NCT02065791), DAPA-CKD (NCT03036150) and SCORED (NCT03315143) trials demonstrate benefits of SGLT inhibition for chronic kidney disease, but they are beyond the scope of this review. ${ }^{53-58}$

Given the dramatic evidence obtained thus far supporting the benefit of SGLT inhibitors in HFrEF, this class of medications must be incorporated into a new backbone of GDMT. The magnitude of benefit of HF interventions can be understood by comparing the relative risk reduction (RRR) of all-cause mortality and HF hospitalizations in landmark clinical trials (Figure 2). ${ }^{59-68}$ In terms of mortality benefit, hydralazine and isosorbide dinitrate showed a 43\% RRR in treatment-naïve patients, beta blockers 34\%, ACE-I/ARB 17\% and MRA 30\%. ${ }^{59}$ More recent medical and device therapies expect patients with HF to be on GDMT with at least beta blockers and ACE-I/ARB for their trials. With this in mind, ARNIs have displayed an impressive additional $14 \%$ RRR in mortality, ICDs 29\%, and CRT 22\%.60-62 With such significant cumulative mortality benefit, contemporary medications have only displayed RRR in HF hospitalizations, such as a $23 \%$ RRR with ivabradine and $8 \%$ with vericiguat. ${ }^{64,65}$ Despite all of the benefit derived from these therapies, SGLT2 inhibitors, using the landmark DAPA-HF trial, present a dramatic further mortality RRR of $16 \%$ and $\mathrm{HF}$ hospitalization RRR of $27 \% .{ }^{41}$

With an extremely favourable risk-benefit ratio, SGLT2 inhibitors should be among the first medications initiated for patients. In the recently published 2021 update to the 2017 American College of Cardiology Expert Consensus Decision Pathway for Optimization of Heart Failure Treatment, the authors suggest adding SGLT2 inhibitors in conjunction with beta blockers, ACE-I/ARB/ARNI (preferred) and MRA in all patients with HFrEF (ejection fraction $<40 \%$ ) except those with contraindications: type 1 diabetes, dialysis, lactation and known hypersensitivity to the medication. ${ }^{69}$ While many of the adverse effects of using SGLT2 inhibitors are mild and quickly diagnosed, the rare adverse effect of euglycaemic diabetic ketoacidosis does exist and can be fatal due to difficulty in early diagnosis and treatment. Insulinopenia with SGLT2 inhibition of the proximal tubules results in altered kidney ATP utilization and increased glucagon release causing loss of bicarbonate, increased ketogenesis and near-normal glucose levels. ${ }^{70}$ However, with increasing awareness to recognize and mitigate this, the benefits of SGLT2 inhibitor use continue to significantly outweigh the risks. Additionally, SGLT2 inhibitors do not have similar issues, such as hypotension and hyperkalaemia, and are renally protective in chronic kidney disease, arguing that this class of medication is an option for those in whom other GDMT use may be restricted. Going forward, there may also be enough evidence to recommend SGLT2 inhibitors for the management of HFpEF, but that remains to be seen.
1. Heidenreich PA, Albert NM, Allen LA, et al. Forecasting the : a policy statement from the American Heart Association. Circ Heart Fail. 2013;6:606-19.

2. Jackson SL, Tong $X$, King RJ, et al. National burden of heart failure events in the United States, 2006 to 2014. Circ Heart Fail. 2018;11:e004873.

3. Davis RC, Hobbs FD, Lip GY. ABC of heart failure. History and epidemiology. BMJ. 2000;320:39-42.

4. Cohn JN, Archibald DG, Ziesche S, et al. Effect of vasodilator therapy on mortality in chronic congestive heart failure. Results of a veterans Administration Cooperative Study. N Eng/ I Med. 1986;314:1547-52.

5. CONSENSUS Trial Study Group. Effects of enalapril on mortality in severe congestive heart failure. Results of the Cooperative North Scandinavian Enalapril Survival Study (CONSENSUS). North Scandinavian Enalapril Sur
N Eng/ J Med. 1987:316:1429-35.

6. Ferrannini E. Sodium-glucose co-transporters and their inhibition: clinical physiology. Cell Metab. 2017;26:27-38. Wright EM, LOo DD, Hirayama BA. Biology of human sodium glucose transporters. Physiol Rev. 2011;91:733-94.

8. Vrhovac I, Balen Eror D, Klessen D, et al. Localizations of $\mathrm{Na}(+)$ D-glucose cotransporters SGLT1 and SGLT2 in human kidney and of SGLT1 in human small intestine, liver, lung, and heart. Pflugers Arch. 2015;467:1881-98.

9. Mackenzie B, Loo DD, Panayotova-Heiermann M, et al. Biophysical characteristics of the pig kidney $\mathrm{Na}+/$ glucose cotransporter SGLT2 reveal a common mechanism for SGLT1 and SGLT2. J Biol Chem. 1996;271:32678-83.

10. Hediger MA, Rhoads DB. Molecular physiology of sodium-glucose cotransporters. Physiol Rev. 1994 74:993-1026

11. Ehrenkranz JR, Lewis NG, Kahn CR, Roth J. Phlorizin: a review. Diabetes Metab Res Rev. 2005;21:31-8.

12. Muscelli E, Astiarraga B, Barsotti E, et al. Metabolic consequences of acute and chronic empagliflozin administration in treatment-naive and metformin pretreated patients with type 2 diabetes. Diabetologia. 2016;59:700-8.

13. Ferrannini E, Muscelli E, Frascerra S, et al. Metabolic response to sodium-glucose cotransporter 2 inhibition in type 2 diabetic patients. J Clin Invest 2014:124:499-508.

4. Nissen SE, Wolski K. Effect of rosiglitazone on the risk of myocardial infarction and death from cardiovascular causes. N Eng/ J Med. 2007;356:2457-71

15. Goldfine AB. Assessing the cardiovascular safety of diabetes therapies. N Engl J Med. 2008;359:1092-5.

16. Scheen AJ. Cardiovascular effects of new oral glucose-lowering agents: DPP- 4 and SGLT-2 inhibitors. Circ Res. 2018;122:1439-59

17. Grempler R, Thomas $L$, Eckhardt $M$, et al. Empagliflozin, a nove selective sodium glucose cotransporter-2 (SGLT-2) inhibitor: characterisation and comparison with other SGLT-2 inhibitors. Diabetes Obes Metab. 2012;14:83-90.

18. Mudaliar S, Polidori D, Zambrowicz B, et al. Sodium-glucose cotransporter inhibitors: effects on renal and intestinal glucose transport: from bench to bedside. Diabetes Care glucose transport:

19. Zinman B, Wanner C, Lachin JM, et al. Empagliflozin cardiovascular outcomes, and mortality in type 2 diabetes. N Eng/ J Med. 2015;373:2117-28.

20. ClinicalTrials.gov. Bl 10773 (empagliflozin) cardiovascular outcome event trial in type 2 diabetes mellitus patients (EMPA-REG OUTCOME). ClinicalTrials.gov Identifier: NCT01131676. Available at: https://clinicaltrials.gov/ct2/show/ NCT01131676 (accessed 22 June 2021).

21. Neal B, Perkovic V, Mahaffey KW, et al. Canagliflozin and cardiovascular and renal events in type 2 diabetes. NEng J Med. 2017;377:644-57.

22. ClinicalTrials.gov. CANVAS - canagliflozin cardiovascular assessment study (CANVAS). ClinicalTrials. gov Identifier: assessment study (CANVAS). ClinicalTrials.gov Identifier:
NCT01032629. Available at: https://clinicaltrials.gov/ct2/show/ NCT01032629 (accessed 22 June 2021)

23. ClinicalTrials gov A study of the effects of canagliflozin (JNJ-28431754) on renal endpoints in adult participants with type 2 diabetes mellitus (CANVAS-R). ClinicalTrials.gov Identifie NCT01989754. Available at: https://clinicaltrials.gov/ct2/show/ NCT01989754 (accessed 22 June 2021).

24. Wiviott SD, Raz I, Bonaca MP, et al. Dapagliflozin and cardiovascular outcomes in type 2 diabetes. N Eng/ J Med. 2019;380:347-57
25. ClinicalTrials gov Multicenter trial to evaluate the effect of dapagliflozin on the incidence of cardiovascular events (DECLARE-TIMI58). ClinicalTrials.gov Identifier: NCT01730534. Available at: https://clinicaltrials.gov/ct2/show/NCT01730534 (accessed 22 June 2021).

26. Cannon CP, Pratley R, Dagogo-Jack S, et al. Cardiovascular outcomes with ertugliflozin in type 2 diabetes. N Eng/ I Med. 2020;383:1425-35.

27. ClinicalTrials.gov. Cardiovascular outcomes following ertugliflozin treatment in type 2 diabetes mellitus participants with vascular disease, the VERTIS CV study (MK-8835-004). ClinicalTrials.gov Identifier: NCT01986881. Available at: https://clinicaltrials.gov/ct2/show/NCT01986881 (accessed 22 June 2021).

28. ClinicalTrials.gov. Specified drug use results survey of Ipragliflozin treatment in type 2 diabetes patients (STELLALONGTERM). ClinicalTrials.gov Identifier: NCT02479399. Available at: https://clinicaltrials.gov/ct2/show/NCT02479399 (accessed 15 June 2021).

29. ClinicalTrials.gov. The clinical study to assess the effect of the amount of carbohydrate intake and meals differing in glycemic index (GI) in patients treated with a sodium-dependent glucose cotransporter 2 (SGLT2) inhibitor. Clinical Trials.gov Identifier: NCT02500186. Available at: https://clinicaltrials.gov/ct2/show/ NCT02500186 (accessed 15 June 2021).

30. ClinicalTrials.gov. TOFO insulin combination trial. ClinicalTrials gov Identifier: NCT02201004. Available at: https://clinicaltrials. gov/ct2/show/NCT02201004 (accessed 15 June 2021).

31. ClinicalTrials.gov. Safety and efficacy of biphasic remogliflozin etabonate in the treatment of type 2 diabetes (BRID). ClinicalTrials.gov Identifier: NCT02537470. Available at: https://clinicaltrials.gov/ct2/show/NCT02537470 (accessed https://Clinicaltic

32. Pereria MJ, Eriksson JW. Emerging role of SGLT-2 inhibitors for the treatment of obesity. Drugs. 2019;79:219-30.

33. Sato T, Aizawa Y, Yuasa S, et al. The effect of dapagliflozin treatment on epicardial adipose tissue volume. Cardiovasc Diabetol. 2018;17:6.

34. Riehle $C$, Abel ED. Insulin signaling and heart failure. Circ Res. 2016;118:1151-69 
35. Omar M, Jensen J, Frederiksen PH, et al. Effect of empagliflozin on hemodynamics in patients with heart failure and reduced ejection fraction. J Am Coll Cardiol. 2020;76:2740-51.

36. Marton A, Kaneko T, Kovalik JP, et al. Organ protection by SGLT2 inhibitors: role of metabolic energy and water conservation. Nat Rev Nephrol. 2021;17:65-77.

37. Sano M, Takei M, Shiraishi Y, et al. Increased hematocrit during sodium-glucose cotransporter 2 inhibitor therapy indicates recovery of tubulointerstitial function in diabetic kidneys. J Clin Med Res. 2016;8:844-7.

38. Chino Y, Samukawa Y, Sakai S, et al. SGLT2 inhibitor lowers serum uric acid through alteration of uric acid transport activity in renal tubule by increased glycosuria. Biopharm Drug Dispos. 2014;35:391-404.

39. Bosch A, Ott C, Jung S, et al. How does empagliflozin improve arterial stiffness in patients with type 2 diabetes mellitus? Sub analysis of a clinical trial. Cardiovasc Diabetol. 2019;18:44.

40. Li C, Zhang J, Xue M, et al. SGLT2 inhibition with empagliflozin attenuates myocardial oxidative stress and fibrosis in diabetic mice heart. Cardiovasc Diabetol. 2019;18:15

41. MCMurray JJV, Solomon SD, Inzucchi SE, et al. Dapagliflozin in patients with heart failure and reduced ejection fraction. N Engl J Med. 2019;381:1995-2008.

42. ClinicalTrials.gov. Study to evaluate the effect of dapagliflozin on the incidence of worsening heart failure or cardiovascular death in patients with chronic heart failure (DAPA-HF). ClinicalTrials.gov Identifier: NCT03036124. Available at: https://clinicaltrials.gov/ct2/show/NCT03036124 (accessed 22 June 2021).

43. Packer $\mathrm{M}$, Anker SD, Butler J, et al. Cardiovascular and renal outcomes with empagliflozin in heart failure. N Eng/ / Med. 2020:383:1413-24.

44. ClinicalTrials.gov. Empagliflozin outcome trial in patients with chronic heart failure with preserved ejection fraction with chronic heart fallure with preserved ejection
(EMPEROR-Preserved). ClinicalTrials.gov Identifier: NCT03057951. Available at: https://clinicaltrials. gov/ct2/show/ NCT03057951. Available at: https://clinicat
NCT03057951 (accessed 22 June 2021)

45. Bhatt DL, Szarek M, Steg PG, et al. Sotagliflozin in patients with Bhatt DL, Szarek M, Steg PG, et al. Sotagliflozin in patients
diabetes and recent worsening heart failure. N Eng/ I Med. 2021;384:117-28.

46. ClinicalTrials. gov. Effect of sotagliflozin on cardiovascular events in patients with type 2 diabetes post worsening heart failure (SOLOIST-WHF Trial). ClinicalTrials.gov Identifier: NCT03521934. Available at: https://clinicaltrials.gov/ct2/show/ NCT03521934 (accessed 15 June 2021).

47. Nassif ME, Windsor SL, Tang F, et al. Dapagliflozin effects on biomarkers, symptoms, and functional status in patients with heart failure with reduced ejection fraction the DEFINE-HF trial. Circulation. 2019;140:1463-76.

48. ClinicalTrials.gov. Are the "Cardiac Benefits" of Empagliflozin Independent of Its Hypoglycemic Activity? (ATRU-4). (EMPA-TROPISM). ClinicalTrials.gov Identifier: NCT03485222. Available at: https://clinicaltrials.gov/ct2/show/NCT03485222 (accessed 22 June 2021).

49. Santos-Gallego $\mathrm{CG}$, Vargas-Delgado AP, Requena-Ibanez JA, et al. Randomized trial of empagliflozin in nondiabetic patients with heart failure and reduced ejection fraction. J Am Coll Cardiol. 2021;77:243-55.

50. Lee MMY, Brooksbank KJM, Wetherall K, et al. Effect of empagliflozin on left ventricular volumes in patients with type 2 diabetes, or prediabetes, and heart failure with reduced ejection fraction (SUGAR-DM-HF). Circulation. 2021;143:516-25.

51. ClinicalTrials.gov. Studies of empagliflozin and its cardiovascular, renal and metabolic effects (SUGAR-DM-HF). ClinicalTrials.gov Identifier: NCT03485092. Available at: https://clinicaltrials.gov/ct2/show/NCT03485092 (accessed 22 June 2021)

52. ClinicalTrials.gov. Dapagliflozin evaluation to improve the lives of patients with preserved ejection fraction heart failure (DELIVER). ClinicalTrials.gov Identifier: NCT03619213. Available at: https: //clinicaltrials gov/ct2/show/NCT03619213 (accessed 15 June 2021).

53. Perkovic V, Jardine MJ, Neal B, et al. Canagliflozin and renal outcomes in type 2 diabetes and nephropathy. N Eng/ J Med. 2019;380:2295-306.

54. ClinicalTrials.gov. Evaluation of the effects of canagliflozin diabetic nephropathy (CREDENCE). ClinicalTrials gov with NCT02065791. Available at: https://clinicaltrials.gov/ct2/show/ NCT02065791 (accessed 15 June 2021).

55. Heerspink HJ, Stefánsson BV, Correa-Rotter R, et al, Dapagliflozin in patients with chronic kidney disease. N Engl I Med 2020:383:1436-46.

56. ClinicalTrials.gov. A study to evaluate the effect of dapagliflozin on renal outcomes and cardiovascular mortality in patients with chronic kidney disease (Dapa-CKD). ClinicalTrials.gov Identifier: NCT03036150. Available at: https://clinicaltrials.gov/ ct2/show/NCT03036150 (accessed 15 June 2021).

57. Bhatt DL, Szarek M, Pitt B, et al. Sotagliflozin in patients with diabetes and chronic kidney disease. N Eng/ J Med. 2021;384:129-39

58. ClinicalTrials.gov. Effect of sotagliflozin on cardiovascular and renal events in patients with type 2 diabetes and moderate renal impairment who are at cardiovascular risk (SCORED). ClinicalTrials.gov Identifier: NCT03315143. Available at:
https://clinicaltrials.gov/ct2/show/NCT03315143 (accessed 15 June 2021).

59. Yancy CW, Jessup M, Bozkurt B, et al. 2013 ACCF/AHA guideline for the management of heart failure: executive summary: a report of the American College of Cardiology Foundation/American Heart Association Task Force on practice guidelines. Circulation. 2013;128:1810-52.

60. McMurray JJ, Packer M, Desai AS, et al. Angiotensin-neprilysin inhibition versus enalapril in heart failure. $N$ Eng/ I Med. 2014;371:993-1004.

61. Moss AJ, Zareba W, Hall WJ, et al. Prophylactic implantation of a defibrillator in patients with myocardial infarction and reduced ejection fraction. N Eng/ J Med. 2002;346:877-83.

62. Bristow MR, Saxon LA, Boehmer J, et al. Cardiac-resynchronization therapy with or without an implantable defibrillator in advanced chronic heart failure N Eng/ J Med. 2004;350:2140-50.

63. Anand IS, Carson P, Galle E, et al. Cardiac resynchronization therapy reduces the risk of hospitalizations in patients with advanced heart failure: results from the comparison of advanced heart failure: results from the Comparison of Medical Therapy, Pacing and Defibrillation in Heart

64. Swedberg K, Komajda M, Böhm M, et al. Ivabradine and outcomes in chronic heart failure (SHIFT): a randomised placebo-controlled study. Lancet. 2010 376:875-85.

65. Armstrong PW, Pieske B, Anstrom KJ, et al. Vericiguat in patients with heart failure and reduced ejection fraction. N Eng/ J Med. 2020;382:1883-93.

66. Digitalis Investigation Group. The effect of digoxin on mortality and morbidity in patients with heart failure. N Eng/ J Med. 1997;336:525-33.

67. Ponikowski P, van Veldhuisen DJ, Comin-Colet J, et al. Beneficial effects of long-term intravenous iron therapy with ferric carboxymaltose in patients with symptomatic heart ferric carboxymaltose in patients with symptomatic heart

68. Teerlink JR, Diaz R, Felker GM, et al. Cardiac myosin activation with omecamtiv mecarbil in systolic heart failure. N Eng/ J Med. 2021;384:105-16.

69. Maddox TM, Januzzi JL Jr, Allen LA, et al. 2021 update to the 2017 ACC Expert Consensus Decision Pathway for Optimization of Heart Failure Treatment: answers to 10 pivotal issues about heart failure with reduced ejection fraction: A report of the American College of Cardiology Solution Set Oversight Committee. J Am Coll Cardiol. 2021;77:772-810

70. Palmer BF, Clegg DJ. Euglycemic ketoacidosis as a complication of SGLT2 inhibitor therapy. Clin J Am Soc Nephrol. 2021:CJN.17621120. 